\title{
Multidirectional Product Support System for Decision Making in Textile Industry using Collaborative Filtering Methods
}

\author{
A.Senthil Kumar \\ Research Scholar, \\ Manonmaniam Sundaranar University, \\ Tirunelveli, India
}

\author{
V.MURALI BHASKARAN \\ Principal, \\ Dhirajlal Gandhi College of Technology, Salem, \\ India
}

\begin{abstract}
In the information technology ground people are using various tools and software for their official use and personal reasons. Nowadays people are worrying to choose data accessing and extraction tools and at the time of buying and selling their products and in addition worry about various quality factors such as price, durability, color, size, and availability of the product. The main purpose of the research study is to find solutions to the unsolved existing problems. The proposed algorithm is Multidirectional Rank Prediction (MDRP) decision making algorithm in order to take an effective strategic decision at all the levels of data extraction, used a real time textile dataset and analyzed the results. Finally the results were obtained and compared with the existing measurement methods such as PCC, SLCF, and VSS. The result accuracy is higher than the existing rank prediction methods.
\end{abstract}

\section{Keywords}

Knowledge Discovery in database (KDD), Multidirectional Rank Prediction (MDRP), Pearson's Correlation Coefficient (PCC), VSS (Vector Space Similarity)

\section{INTRODUCTION}

Collaborative techniques are used to filter the noise data and give product recommendation to the new users. Collaborative filtering technique may involve in large data sets. Collaborative technique is used to predict user's interests on particular product or more than one product. In this Proposal, Selling and buying are the two usual activities for seller and buyer. One who sells the products aims to gain maximum earnings, and the customer side to get trustworthy product, further it is extended to intermediate level. These scenarios were identified and people who are using products are found difficulties to discover the good product. In addition people suffer and face problems to identify the features of a product such as color, size, price, availability and durability of the concerned product.

To overcome these situations, identified a technique multidirectional rank prediction similarity collaborative filtering. Normally Collaborative filtering algorithms are used to find users' dynamic participation, a simple way to represent users' interests to the system, and finally the algorithm which is used to match people with similar interests.
Still data extraction is a primary problem in different disciplines, to handle them and provide solution. Solving such kind of problems using available techniques is a regular method, but the difficulty is to identify a best method or technique for long-term solution to the existing problem.

In the collaborative filtering approach, the recommender system used to identify users who have the same preferences with the current user, and propose items which the user most likes. Due to this cold start problem, this approach was failed to consider items which nobody was rated previously. The collaborative filtering system require a large number of users to rate a new item before that item can be recommended.

In the existing methods are provided with some solutions, but it is not up to the anticipated level to the customers. Hence customers are mostly suffering sparsity and scalability problems while they are using systems are associated with large data sets. The user-item matrix which is used in collaborative filtering could be tremendously large and sparse, which carries out the challenges in the performances of the recommendation.

\subsection{Data Sparsity Problem}

One of the major problems in data sparsity is the cold start problem. As collaborative filtering methods recommend items based[10] on users' past preferences, new users must need to rank adequate number of items which is used to enable the system to capture their preferences accurately and to offer reliable recommendations.

Similarly, the users when rating the new items also face the same problem. While new items are appended to the existing application, they must to be rated by large number of users before they could be recommended to users who have similar tastes with the ones rated them. Rating to the new products is very tough to the users, this type of problem is mainly focused Sometimes the new product will be recommended based on qualities rather than past ratings.

\subsection{Scalability Problem}

The second problem in existing collaborative filtering algorithms is facing scalability. If the customer data set contains huge no. of items, time complexity will be increased and rapid response will not get from those situations. To handle this situation online rating method was introduced and dealt these problems very seriously, but users are rated the products based on the past ratings and 
sometime users could not see the previous ratings due to unavailability in the particular application and lack of time. In the case of big data set, the system has to respond immediately and provide recommendations.

Many Collaborative Filtering algorithms are highlighting only on user similarity and item similarity, and initially they are based on User-defined similarity measurements, such as Pearson Correlation Coefficient (PCC) or Vector Space Similarity (VSS), which are not suitable and optimized for specific applications and large data sets. Next, these similarity measures are limited to symmetric ones such that the similarity between A and B is the same as that for B and A, although symmetry is not hold in many real world applications. Then, next typically take care of the similarity functions between users and functions between items individually. However, in reality, the similarities between users and between items are inter-related. In the earlier article, identified a unified model for users and items, known as Similarity Learning based Collaborative Filtering (SLCF), based on a bidirectional asymmetric similarity measurement. The above SLCF and R-SLCF methods are not deals with user biography, classification of customer based on time, location, and brand with multiple entities.

\subsection{Limitations of Uni-Directional and Bi- Directional Similarity Learning Based Collaborative Filtering Techniques (SLCF \&R-SLCF)}

SLCF \& R-SLCF model[1] automatically finds out the asymmetric similarities between users and items at a time through matrix factorization technique and which includes a new matrix factorization model for learning user and item similarities simultaneously. In addition similarity measurement was asymmetric and can be identified from the data using matrix factorization methods. This earlier SLCF method was not effective to find prediction ratings because which not includes the past ratings, user biography and classification etc. SLCF similarity measurement was considerably satisfied but it is to be redefined. The experimental results of SLCF were focused only on compared with previous item based and user based in previous traditional memory-based approaches and a lowrank matrix approximation models. But, it is not an appropriate model to deal with multiple entities. Additionally, the online version of the rank prediction algorithm is exposed to be efficient and more capable for handling new users and items. So, here proposed an online version of rate prediction method to integrate new users and new items in a successful way. Moreover, a plan to develop an added efficient algorithms to learn more models for larger scale datasets.

An earlier model SLCF \& R-SLCF was very general for sparse data and scalability problems. Therefore, the earlier bidirectional model were used towards the following benchmark datasets, which includes Movie Lens, Netflix Movie Data Sets, and Technlens+ datasets but, the SLCF \& R-SLCF models are inappropriate for textile data set, garment related and web mining applications.

\subsection{Multidirectional Asymmetric Similarity Learning}

To extend this proposed model to multi-directional cases where more than two types of entities are involved such as user, item, ratings, seller (either manufacturer or supplier).In the multidirectional asymmetric similarity learning algorithm mainly focuses on item-item similarity and useruser similarity simultaneously, to create two connected contributions. The first contribution is similarity function learning. Next contribution is proposed a unified model to learn asymmetric similarities for items and users at the same time.

Various hybrid algorithms were used for data extraction some hybrid algorithms are appropriate only for some scenarios like such a situation user's privacy should be protected while the users are rating an item. In few circumstances item-item similarity is better than the useruser similarity while hiding the user demographic information. Existing PCC (Pearson Correlation Coefficient and VSS (Vector Space Similarity) is inappropriate for all type of applications. To protect the privacy[6] of users cryptographical algorithms are used because item-item similarity is publicly available. But the major drawback of this algorithm was item-user similarity to be calculated parallel. The objective of the content based filtering and collaborative filtering was focused on data set to be reduced and this is not suitable for all kinds of applications

Considered the two earlier versions of SLCFs, the first one was improving the traditional memory-based approaches (M-SLCF), and second one was based on matrix reconstruction (R-SLCF). But the earlier proposed online version of the rating prediction method R-SLCF to allow new users and new items to be included in the model incrementally but with no classifications and not to use for larger scale datasets. The MDRP model is handling sparse and scalability problems in an efficient and effective way. Therefore, using the proposed model to other kinds of data sets such as journal citation data set, internet usage tracking and job applications web portals. So that, the modified MDRP CF will be performing very well in case of item to user relationship in multiple ways.

Finally need to have an optimized rank prediction algorithmic technique to solve sparcity, scalability, classification, and measurement problems. In this paper MDRP algorithm can be used to solve all the above mentioned problems and additionally it deals with classifying the items based on person, brand and quality factors, and visualized the data in different forms. Finally with respect to different quality constraints analyzed the data set with performance measurements on the basis of which users can take suitable decisions.

\section{MULTIDIRECTIONAL DECISION MAKING RANK PREDICTION ALGORITHM (MDRP)}

To overcome all the exiting problems at various user levels, using Multidirectional Rank Prediction (MDRP) Decision Making Algorithm. In this proposed model is associated 
with multidirectional data set with multiple entities with more than two attributes are concerned.

Table 1: Multidirectional Entities with Multiple Attributes and Scaling

\begin{tabular}{|c|c|c|c|c|c|}
\hline Entities & $\mathbf{r}$ & Age & rofes & Location & Scaling \\
\hline & \multirow{4}{*}{$\begin{array}{l}\text { Male, } \\
\text { Female }\end{array}$} & \multirow{4}{*}{$\begin{array}{c}17-20 \\
21-45 \\
\text { Above } \\
45\end{array}$} & \multirow{4}{*}{$\begin{array}{c}\text { Teacher, } \\
\text { Engineer, } \\
\text { Business } \\
\text { person, } \\
\text { Doctor etc. }\end{array}$} & \multirow{4}{*}{$\begin{array}{l}\text { Urban, } \\
\text { Semi- } \\
\text { urban, } \\
\text { Rural. }\end{array}$} & \multirow{4}{*}{$\begin{array}{l}5 \text { point } \\
\text { Scale } \\
\text { method }\end{array}$} \\
\hline & & & & & \\
\hline Ra & & & & & \\
\hline $\operatorname{Pr}$ & & & & & \\
\hline
\end{tabular}

Initially Weka 3.6.6 tool was chosen and analyzed the experimental results on the basis of data mining functions such as classification, cluster analysis, making associations with different entities, regression analysis and prediction. The multidimensional data set was taken and examined the results in various aspects such as income, location, age group and gender.

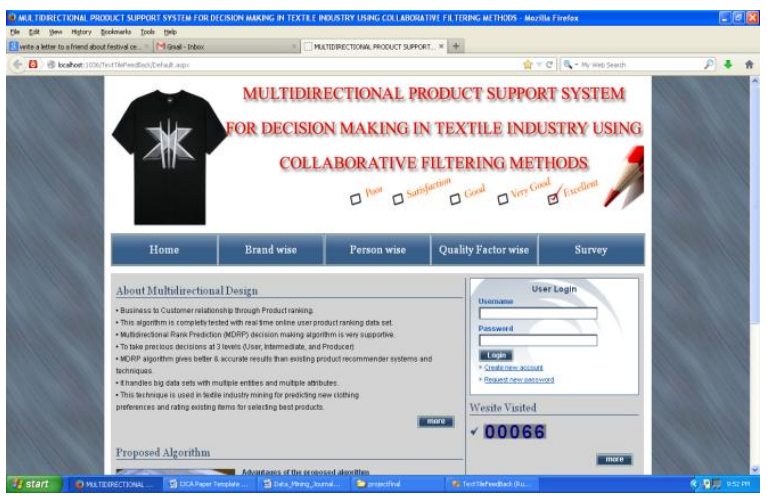

Figure 1: Multidirectional Collaborative Filtering

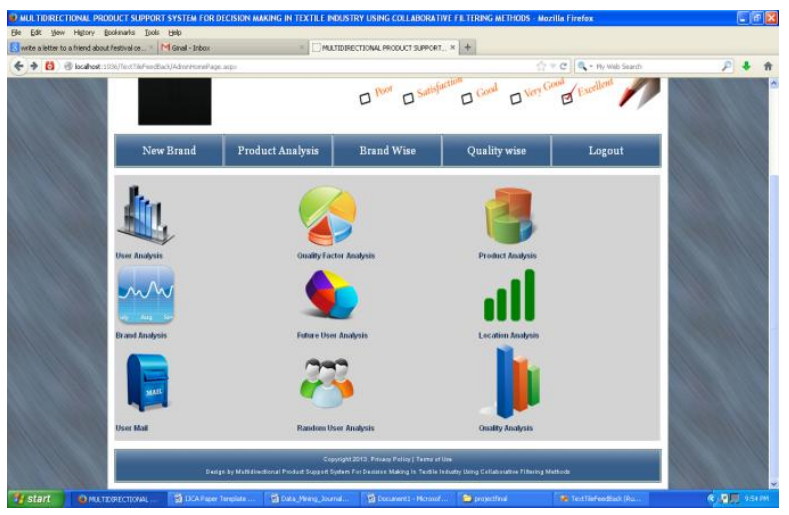

Figure 2: Product Ranking User Survey Analysis

In this section, we considered various parameters such as gender, age group, user category, brand details. Then analyzed experimental results with different combinations and comparisons with different time frame. Mainly users are categorized with different scenarios and prepared various reports for further improvements and enhancements of the current system. This online survey method is very much useful to the new users to rate new items.

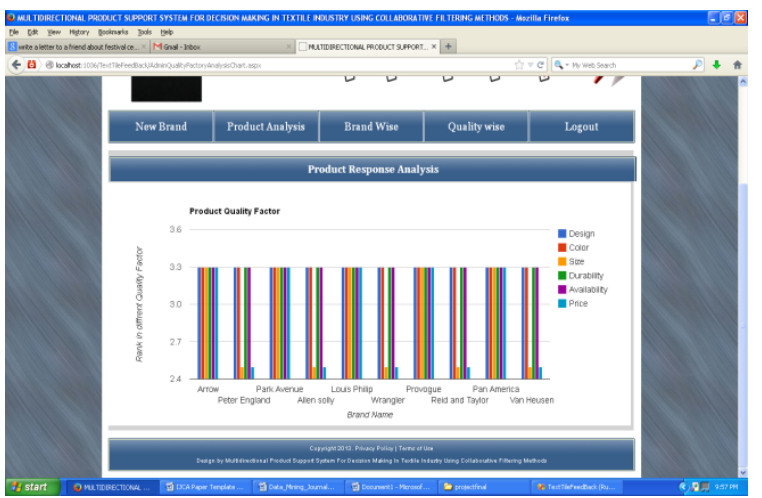

Figure 3: Men's Clothing Preferences

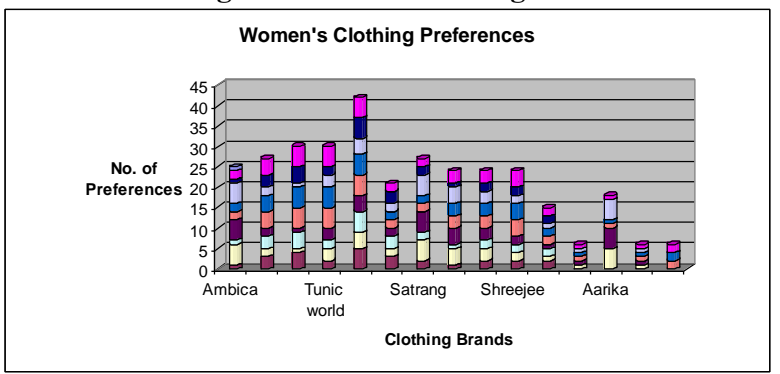

Figure 4: Women's Clothing Preferences

The above graph shows that various brand results which observed from the textile data set and analyzed, compared the results with various products. Ranking of various products by the user and can be used to assess the quality of the product based on the quality constraints and make an effective decision on purchase. This strategic decision making is very valuable to increase the sales.

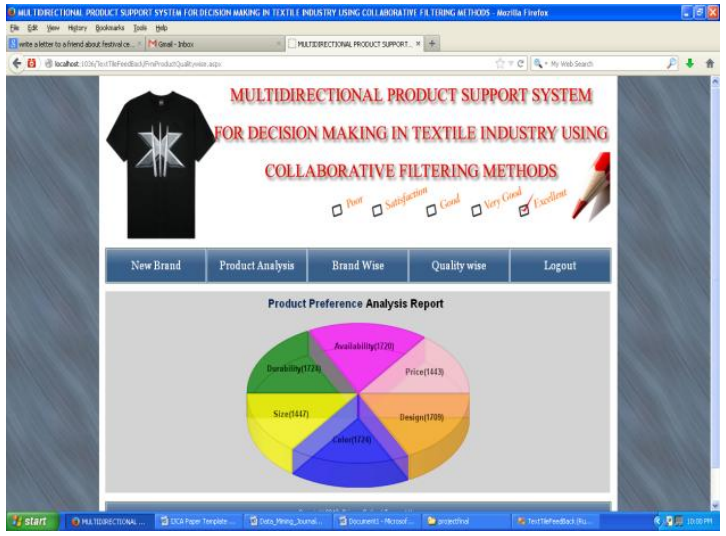

Figure 5: Product Preference Analysis

\section{CONCLUSION}

The above multidirectional online rank prediction algorithm helps to find the right product at the right time of purchase and analyze the existing user purchase history. The proposed MDRP algorithm is very supportive to make high-quality decisions at three steps position levels. It gives accurate results over than SLCF, RLCF, PCC and VSS measurement 
methods. It handles large data sets with multiple entities, multiple attributes feasibly. Right product can survive in the market based on the good product rating. Low ranked products will be eliminated or to be improved. Customer can get chance to buy good product with good features.

\section{REFERENCES}

[1] Learning bidirectional asymmetric similarity for collaborative filtering via matrix factorization Zheng Chen,Bin Cao,Qiang Yang,Jian-Rao Sun.Springer, Data Mining and Knowledge Discovery (2011) 22:393-418.

[2] Adomavicius G, Tuzhilin A (2005) Toward the next generation of recommender systems: a survey of the state-of-the-art and possible extensions. IEEE TKDE 17(6):734-749.

[3] Almeida LB, Langlois T, Amaral JD, Plakhov A (1998) Parameter adaptation in stochastic optimization.In: Saad D (ed) On-line learning in neural networks. Cambridge University Press, Cambridge, pp 111-134

[4] BrandM(2003) Fast online SVD revisions for lightweight recommender systems. In: Proceedings of SIAMICDM
[5] Breese JS, Heckerman D, Kadie C (1998) Empirical analysis of predictive algorithms for collaborative filtering. In: Proceedings of the 14th conference on UAI, pp 43-52

[6] Canny J (2002) Collaborative filtering with privacy via factor analysis. In: Proceedings of the 25th SIGIR. ACM, New York, pp 238-245

[7] Cao B, Sun J-T, Wu J, Yang Q, Chen Z (2008) Learning bidirectional similarity for collaborative filtering. In: Proceedings of the ECML/PKDD, part 1, pp 178-194

[8] Chen G, Wang F, Zhang C (2009) Collaborative filtering using orthogonal nonnegative matrix trifactorization. Inf Process Manag 45(3):368-379.

[9] Delgado J (1999) Memory-based weighted majority prediction for recommender systems.In: ACM SIGIR'99 workshop on recommender systems.Deshpande M, Karypis G (2004) Item-based top-n recommendation algorithms. ACM TOIS 22(1): 143-177.

[10] Deshpande M, Karypis G (2004) Item-based top-n recommendation algorithms. ACM TOIS 22(1): 143177. 